PROCEEDINGS OF THE

AMERICAN MATHEMATICAL SOCIETY

Volume 137, Number 9, September 2009, Pages 3155-3161

S 0002-9939(09)09908-0

Article electronically published on May 4, 2009

\title{
QUANTUM ISOMETRY GROUP OF THE $n$-TORI
}

\author{
JYOTISHMAN BHOWMICK
}

(Communicated by Varghese Mathai)

\begin{abstract}
We show that the quantum isometry group (introduced by Goswami) of the $n$-tori $\mathbb{T}^{n}$ coincides with its classical isometry group; i.e. there does not exist any faithful isometric action on $\mathbb{T}^{n}$ by a genuine (noncommutative as a $C^{*}$-algebra) compact quantum group. Moreover, using an earlier result, we conclude that the quantum isometry group of the noncommutative $n$ tori is a Rieffel deformation of the quantum isometry group of the commutative $n$-tori.
\end{abstract}

\section{INTRODUCTION}

In 4], Goswami has defined the quantum isometry group of a noncommutative manifold (given by a spectral triple), motivated by the definition and study of quantum permutation groups of finite sets and finite graphs by a number of mathematicians (see, e.g., 1 1, 2, [6, and the references therein) and using some ideas of Woronowicz and Soltan (see [10]).

The main ingredient of this theory is the Laplacian $\mathcal{L}$ coming from the spectral triple $\left(\mathcal{A}^{\infty}, \mathcal{H}, D\right)$ satisfying certain regularity conditions (see [4 for its construction), which coincides with the Hodge Laplacian $-d^{*} d$ (restricted on a space of smooth functions) in the classical case, where $d$ denotes the de Rham differential.

The linear span of eigenvectors of $\mathcal{L}$, which is a subspace of $\mathcal{A}^{\infty}$, is denoted by $\mathcal{A}_{0}^{\infty}$, and it is assumed that $\mathcal{A}_{0}^{\infty}$ is norm-dense in the $C^{*}$-algebra $\mathcal{A}$ obtained by completing $\mathcal{A}^{\infty}$. The $*$-subalgebra of $\mathcal{A}^{\infty}$ generated by $\mathcal{A}_{0}^{\infty}$ is denoted by $\mathcal{A}_{0}$. Then $\mathcal{L}\left(\mathcal{A}_{0}^{\infty}\right) \subseteq \mathcal{A}_{0}^{\infty}$, and a compact quantum group $(\mathcal{G}, \Delta)$ which has an action $\alpha$ on $\mathcal{A}$ is said to act smoothly and isometrically on the noncommutative manifold $\left(\mathcal{A}^{\infty}, \mathcal{H}, D\right)$ if for every state $\phi$ on $\mathcal{G},($ id $\otimes \phi) \circ \alpha\left(\mathcal{A}_{0}^{\infty}\right) \subseteq \mathcal{A}_{0}^{\infty}$ for all states $\phi$ on $\mathcal{G}$, and also (id $\otimes \phi$ ) $\circ \alpha$ commutes with $\mathcal{L}$ on $\mathcal{A}_{0}^{\infty}$.

One can then consider the category of all compact quantum groups acting smoothly and isometrically on $\mathcal{A}$, where the morphisms are quantum group morphisms which intertwine the actions on $\mathcal{A}$. It is proved in [4] (under some regularity assumptions, which are valid for any compact connected Riemannian spin manifold with the usual Dirac operator) that there exists a universal object in this category, and this universal object is defined to be the quantum isometry group of $\left(\mathcal{A}^{\infty}, \mathcal{H}, D\right)$, denoted by $Q I S O\left(\mathcal{A}^{\infty}, \mathcal{H}, D\right)$, or simply as $Q I S O\left(\mathcal{A}^{\infty}\right)$ or even $Q I S O(\mathcal{A})$ if the spectral triple is understood.

Received by the editors May 6, 2008.

2000 Mathematics Subject Classification. Primary 58B32; Secondary 16W30, 46L87, 46L89.

Support from the National Board of Higher Mathematics, India, is gratefully acknowledged.

(C)2009 American Mathematical Society

Reverts to public domain 28 years from publication 
It is important to explicitly describe quantum isometry groups of sufficiently many classical and noncommutative manifolds. In [5], the quantum isometry group of the two-torus has been explicitly computed.

Although there are similarities between the basic principle of the computation of [5] and that of the present article, which is to exploit the eigenspaces of the Laplacian, there is a key difference between the two proofs, which is as follows.

The computation of the quantum isometry group of $\mathcal{A}_{\theta}$ in Section 2.3 of [ [5] is valid for all $\theta$; i.e. have all $\theta$ has been treated on the same footing. On the other hand, we have adopted a different strategy in the present article based on the observation that the proof of [5] can be simplified quite a bit for the special case of $\theta=0$, i.e. the commutative case, and this simplification even goes through easily for any $n \geq 3$. Thus, in this article we first compute the quantum isometry group of the commutative $C^{*}$-algebra $C\left(\mathbb{T}^{n}\right)$. This commutativity assumption decreases a lot of computations because it suffices to show that the quantum isometry group is a commutative $C^{*}$-algebra and hence has to coincide with the classical isometry group. Then the structure of the quantum isometry group of $\mathbb{T}_{\theta}^{n}$ for an arbitrary value of $\theta$ follows immediately from Theorem 3.13 of [5].

Throughout the paper, we have denoted by $\mathcal{A}_{1} \otimes \mathcal{A}_{2}$ the minimal (injective) $C^{*}$-tensor product between two $C^{*}$-algebras $\mathcal{A}_{1}$ and $\mathcal{A}_{2}$. The symbol $\otimes_{\text {alg }}$ has been used to denote the algebraic tensor product between vector spaces or algebras.

For a compact quantum group $\mathcal{G}$, let the dense unital $*$-subalgebra generated by the matrix coefficients of irreducible unitary representations be denoted by $\mathcal{G}_{0}$. The coproduct of $\mathcal{G}$, say $\Delta$, maps $\mathcal{G}_{0}$ into the algebraic tensor product $\mathcal{G}_{0} \otimes_{\text {alg }} \mathcal{G}_{0}$, and there exist a canonical antipode and a counit defined on $\mathcal{G}_{0}$ which make it into a Hopf $*$-algebra (see [7] for the details).

Remark 1.1. In [4, it was assumed that the compact quantum groups are separable. But in [9], the separability assumption for the $C^{*}$-algebra of the underlying compact quantum group was removed. It can be easily seen that the separability was not at all used in the proofs of [4, and hence all the results in [4] proceed verbatim in the nonseparable case.

1.1. Quantum isometry group of the commutative $n$-tori. Consider $C\left(\mathbb{T}^{n}\right)$ as the universal commutative $C^{*}$-algebra generated by $n$ commuting unitaries $U_{1}, U_{2}, \ldots, U_{n}$. It is clear that the set $\left\{U_{i}^{m} U_{j}^{n}: m, n \in \mathbb{Z}\right\}$ is an orthonormal basis for $L^{2}\left(C\left(\mathbb{T}^{n}\right), \tau\right)$, where $\tau$ denotes the unique faithful normalized trace on $C\left(\mathbb{T}^{n}\right)$ given by $\tau\left(\sum a_{m n} U_{i}^{m} U_{j}^{n}\right)=a_{00}$, which is just the integration against the Haar measure. We shall denote by $\langle A, B\rangle=\tau\left(A^{*} B\right)$ the inner product on $\mathcal{H}_{0}:=L^{2}\left(C\left(\mathbb{T}^{n}\right), \tau\right)$. Let $C\left(\mathbb{T}^{n}\right)^{\text {fin }}$ be the unital $*$-subalgebra generated by finite complex linear combinations of $U^{m} V^{n}, m, n \in \mathbb{Z}$. The Laplacian $\mathcal{L}$ is given by $\mathcal{L}\left(U_{1}^{m_{1}}, \ldots, U_{n}^{m_{n}}\right)=-\left(m_{1}^{2}+\ldots m_{n}^{2}\right) U_{1}^{m_{1}}, \ldots, U_{n}^{m_{n}}$, and it is also easy to see that the algebraic span of eigenvectors of $\mathcal{L}$ is nothing but the space $C\left(\mathbb{T}^{n}\right)^{\text {fin }}$ and, moreover, all the assumptions in [4] required for defining the quantum isometry group are satisfied.

Let $\mathcal{Q}$ be the quantum isometry group coming from the above Laplacian, with the smooth isometric action of $\mathcal{Q}$ on $C\left(\mathbb{T}^{n}\right)$ given by $\alpha: C\left(\mathbb{T}^{n}\right) \rightarrow C\left(\mathbb{T}^{n}\right) \otimes \mathcal{Q}$. By definition, $\alpha$ must keep invariant the eigenspace of $\mathcal{L}$ corresponding to the eigenvalue 
-1 , spanned by $U_{1}, \ldots, U_{n}, U_{1}^{-1}, \ldots, U_{n}^{-1}$. Thus, the action $\alpha$ is given by

$$
\alpha\left(U_{i}\right)=\sum_{j=1}^{n} U_{j} \otimes A_{i j}+\sum_{j=1}^{n} U_{j}^{-1} \otimes B_{i j},
$$

where $A_{i j}, B_{i j} \in \mathcal{Q}, i, j=1,2, \ldots, n$. By faithfulness of the action of the quantum isometry group (see [4), the norm-closure of the unital $*$-algebra generated by $A_{i j}, B_{i j} ; i, j=1,2, \ldots, n$ must be the whole of $\mathcal{Q}$.

Next we derive a number of conditions on $A_{i j}, B_{i j}, i, j=1,2, \ldots n$ using the fact that $\alpha$ is a $*$-homomorphism.

Lemma 1.2. The condition $U^{*} U=1=U U^{*}$ gives:

$$
\begin{gathered}
\sum_{j}\left(A_{i j}^{*} A_{i j}+B_{i j}^{*} B_{i j}\right)=1 \\
B_{i j}^{*} A_{i k}+B_{i k}^{*} A_{i j}=0 \forall j \neq k \\
A_{i j}^{*} B_{i k}+A_{i k}^{*} B_{i j}=0 \forall j \neq k \\
A_{i j}^{*} B_{i j}=B_{i j}^{*} A_{i j}=0 \\
\sum_{j}\left(A_{i j} A_{i j}^{*}+B_{i j} B_{i j}^{*}\right)=1 \\
A_{i k} B_{i j}^{*}+A_{i j} B_{i k}^{*}=0 \forall j \neq k \\
B_{i k} A_{i j}^{*}+B_{i j} A_{i k}^{*}=0 \forall j \neq k \\
A_{i j} B_{i j}^{*}=B_{i j} A_{i j}^{*}=0 .
\end{gathered}
$$

Proof. We get (11) - (4) by using the condition $U_{i}^{*} U_{i}=1$ along with the fact that $\alpha$ is a homomorphism and then comparing the coefficients of $1, U_{j} U_{k}, U_{j}^{-1} U_{k}^{-1}$ (for $j \neq k), U_{j}^{-2}, U_{k}^{-2}$.

Similarly the condition $U_{i} U_{i}^{*}=1$ gives (5) - (8) .

Now, $\forall i \neq j, U_{i}^{*} U_{j}, U_{i} U_{j}^{*}$ and $U_{i} U_{j}$ belong to the eigenspace of the Laplacian with eigenvalue -2 , while $U_{k}^{2}, U_{k}^{-2}$ belong to the eigenspace corresponding to the eigenvalue -4 . As $\alpha$ preserves the eigenspaces of the Laplacian, the coefficients of $U_{k}^{2}, U_{k}^{-2}$ are zero $\forall k$ in $\alpha\left(U_{i}^{*} U_{j}\right), \alpha\left(U_{i} U_{j}^{*}\right), \alpha\left(U_{i} U_{j}\right) \forall i \neq j$.

We use this observation in the next lemma.

Lemma 1.3. $\forall k$ and $\forall i \neq j$,

$$
\begin{aligned}
& B_{i k}^{*} A_{j k}=A_{i k}^{*} B_{j k}=0 \\
& A_{i k} B_{j k}=B_{i k} A_{j k}^{*}=0 \\
& A_{i k} A_{j k}=B_{i k} B_{j k}=0 .
\end{aligned}
$$

Proof. The equation (9) is obtained from the coefficients of $U_{k}^{2}$ and $U_{k}^{-2}$ in $\alpha\left(U_{i}^{*} U_{j}\right)$, while (10) and (11) are obtained from the same coefficients in $\alpha\left(U_{i} U_{j}^{*}\right)$ and $\alpha\left(U_{i} U_{j}\right)$, respectively.

Now, by Lemma 2.12 in [4 it follows that $\tilde{\alpha}: C\left(\mathbb{T}^{n}\right) \otimes \mathcal{Q} \rightarrow C\left(\mathbb{T}^{n}\right) \otimes \mathcal{Q}$ defined by $\tilde{\alpha}(X \otimes Y)=\alpha(X)(1 \otimes Y)$ extends to a unitary of the Hilbert $\mathcal{Q}$-module $L^{2}\left(C\left(\mathbb{T}^{n}\right), \tau\right) \otimes \mathcal{Q}$ (or in other words, $\alpha$ extends to a unitary representation of $\mathcal{Q}$ on $\left.L^{2}\left(C\left(\mathbb{T}^{n}\right), \tau\right)\right)$. But $\alpha$ keeps $W=\operatorname{Sp}\left\{U_{i}, U_{i}^{*}: 1 \leq i \leq n\right\}$ invariant. So $\alpha$ is a 
unitary representation of $\mathcal{Q}$ on $W$. Hence, the matrix (say $M$ ) corresponding to the $2 n$-dimensional representation of $\mathcal{Q}$ on $W$ is a unitary in $M_{2 n}(\mathcal{Q})$.

From the definition of the action it follows that $M=\left(\begin{array}{cc}A_{i j} & B_{i j}^{*} \\ B_{i j} & A_{i j}^{*}\end{array}\right)$.

Since $M$ is the matrix corresponding to a finite dimensional unitary representation, $\kappa\left(M_{k l}\right)=M_{k l}^{-1}$, where $\kappa$ denotes the antipode of $\mathcal{Q}$ (see [8]).

But $M$ is a unitary, $M^{-1}=M^{*}$. So, $\left(k\left(M_{k l}\right)\right)=\left(\begin{array}{cc}A_{j i}^{*} & B_{j i}^{*} \\ B_{j i} & A_{j i}\end{array}\right)$.

Now we apply the antipode $\kappa$ to get some more relations.

Lemma 1.4. $\forall k$ and $i \neq j$,

$$
A_{k j}^{*} A_{k i}^{*}=B_{k j} B_{k i}=A_{k j}^{*} B_{k i}^{*}=B_{k j} A_{k i}=B_{k j} A_{k i}^{*}=A_{k j} B_{k i}=0 .
$$

Proof. The result follows by applying $\kappa$ on the equations $A_{i k} A_{j k}=B_{i k} B_{j k}=$ $B_{i k}^{*} A_{j k}=A_{i k}^{*} B_{j k}=A_{i k} B_{j k}=B_{i k} A_{j k}^{*}=0$ obtained from Lemma 1.3 .

Lemma 1.5. $A_{l i}$ is a normal partial isometry $\forall l, i$ and hence has the same domain and range.

Proof. From the relation (11) in Lemma 1.2, we have by applying $\kappa, \sum\left(A_{j i}^{*} A_{j i}+\right.$ $\left.B_{j i} B_{j i}^{*}\right)=1$. Applying $A_{l i}$ on the right of this equation, we have

$$
A_{l i}^{*} A_{l i} A_{l i}+\sum_{j \neq l}\left(A_{j i}^{*} A_{j i} A_{l i}+B_{l i} B_{l i}^{*} A_{l i}\right)+\sum_{j \neq l} B_{j i} B_{j i}^{*} A_{l i}=A_{l i} .
$$

From Lemma 1.3, we have $A_{j i} A_{l i}=0$ and $B_{j i}^{*} A_{l i}=0 \forall j \neq l$. Moreover, from Lemma 1.2, we have $B_{l i}^{*} A_{l i}=0$. Applying these to the above equation, we have

$$
A_{l i}^{*} A_{l i} A_{l i}=A_{l i} \text {. }
$$

Again, from the relation $\sum_{j}\left(A_{i j} A_{i j}^{*}+B_{i j} B_{i j}^{*}\right)=1 \forall i$ in Lemma 1.2, applying $\kappa$ and multiplying by $A_{l i}^{*}$ on the right, we have $A_{l i} A_{l i}^{*} A_{l i}^{*}+\sum_{j \neq l} A_{j i} A_{j i}^{*} A_{l i}^{*}+$ $B_{l i}^{*} B_{l i} A_{l i}^{*}+\sum_{j \neq l} B_{j i}^{*} B_{j i} A_{l i}^{*}=A_{l i}^{*}$. From Lemma 1.3, we have $A_{l i} A_{j i}=0 \forall j \neq l$ (hence $A_{j i}^{*} A_{l i}^{*}=0$ ) and $B_{j i} A_{l i}^{*}=0$. Moreover, we have $B_{l i} A_{l i}^{*}=0$ from Lemma 1.2 . Hence, we have

$$
A_{l i} A_{l i}^{*} A_{l i}^{*}=A_{l i}^{*}
$$

From (13), we have

$$
\left(A_{l i}^{*} A_{l i}\right)\left(A_{l i} A_{l i}^{*}\right)=A_{l i} A_{l i}^{*} .
$$

By taking $*$ on (14), we have

$$
A_{l i} A_{l i} A_{l i}^{*}=A_{l i} .
$$

Using this in (15), we have

$$
A_{l i} A_{l i}^{*} A_{l i}=A_{l i} A_{l i}^{*}
$$

and hence $A_{l i}$ is normal.

So, $A_{l i}=A_{l i}^{*} A_{l i} A_{l i}($ from (13) $)=A_{l i} A_{l i}^{*} A_{l i}$.

Therefore, $A_{l i}$ is a partial isometry which is normal and hence has the same domain and range.

Lemma 1.6. $B_{l i}$ is a normal partial isometry and hence has the same domain and range. 
Proof. First we note that $A_{j i}$ is a normal partial isometry and that $A_{j i} B_{l i}=0$ $\forall j \neq l$ (obtained from Lemma 1.3) implies that $\operatorname{Ran}\left(A_{j i}^{*}\right) \subseteq \operatorname{Ker}\left(B_{l i}^{*}\right)$ and hence $\operatorname{Ran}\left(A_{j i}\right) \subseteq \operatorname{Ker}\left(B_{l i}^{*}\right)$, which means that $B_{l i}^{*} A_{j i}=0 \forall j \neq l$.

To obtain $B_{l i}^{*} B_{l i} B_{l i}=B_{l i}$, we apply $\kappa$ and multiply by $B_{l i}$ on the right of (5) and then use $A_{l i}^{*} B_{l i}=0$ from Lemma 1.2, $A_{j i} B_{l i}=0 \forall j \neq l$ (from Lemma 1.3, which implies $B_{l i}^{*} A_{j i}=0 \forall j \neq l$ as above) and $B_{j i} B_{l i}=0 \forall j \neq l$ from Lemma 1.3.

Similarly, we have $B_{l i} B_{l i}^{*} B_{l i}^{*}=B_{l i}^{*}$ by applying $\kappa$ and multiplying by $B_{l i}^{*}$ on the right of (1) obtained from Lemma 1.2] and then using $A_{l i} B_{l i}^{*}=0$ (Lemma 1.2), $B_{l i} A_{j i}^{*}=0 \forall j \neq l$, and $B_{l i} B_{j i}=0 \forall j \neq l$ (Lemma 1.3).

Using $B_{l i}^{*} B_{l i} B_{l i}=B_{l i}$ and $B_{l i} B_{l i}^{*} B_{l i}^{*}=B_{l i}^{*}$ as in Lemma 1.5, we have that $B_{l i}$ is a normal partial isometry.

Now, we use the condition $\alpha\left(U_{i}\right) \alpha\left(U_{j}\right)=\alpha\left(U_{j}\right) \alpha\left(U_{i}\right) \forall i, j$.

Lemma 1.7. $\forall k \neq l$,

$$
\begin{aligned}
& A_{i k} A_{j l}+A_{i l} A_{j k}=A_{j l} A_{i k}+A_{j k} A_{i l} \\
& A_{i k} B_{j l}+B_{i l} A_{j k}=B_{j l} A_{i k}+A_{j k} B_{i l} \\
& B_{i k} A_{j l}+A_{i l} B_{j k}=A_{j l} B_{i k}+B_{j k} A_{i l} \\
& B_{i k} B_{j l}+B_{i l} B_{j k}=B_{j l} B_{i k}+B_{j k} B_{i l} .
\end{aligned}
$$

Proof. The result follows by equating the coefficients of $U_{k} U_{l}, U_{k} U_{l}^{-1}, U_{k}^{-1} U_{l}$ and $U_{k}^{-1} U_{l}^{-1}($ where $k \neq l)$ in $\alpha\left(U_{i}\right) \alpha\left(U_{j}\right)=\alpha\left(U_{j}\right) \alpha\left(U_{i}\right) \forall i, j$.

Lemma 1.8. $A_{i k} B_{j l}=B_{j l} A_{i k} \forall i \neq j, k \neq l$.

Proof. From Lemma 1.7 we have $\forall k \neq l, A_{i k} B_{j l}-B_{j l} A_{i k}=A_{j k} B_{i l}-B_{i l} A_{j k}$. We consider the case where $i \neq j$.

We have $\operatorname{Ran}\left(A_{i k} B_{j l}-B_{j l} A_{i k}\right) \subseteq \operatorname{Ran}\left(A_{i k}\right)+\operatorname{Ran}\left(B_{j l}\right) \subseteq \operatorname{Ran}\left(B_{j l}^{*} B_{j l}+A_{i k}^{*} A_{i k}\right)$ (using the facts that $A_{i k}$ and $B_{j l}$ are normal partial isometries by Lemma 1.5 and 1.6 and also that $B_{j l}^{*} B_{j l}$ and $A_{i k}^{*} A_{i k}$ are projections).

Similarly, $\operatorname{Ran}\left(A_{j k} B_{i l}-B_{i l} A_{j k}\right) \subseteq \operatorname{Ran}\left(B_{i l}^{*} B_{i l}+A_{j k}^{*} A_{j k}\right)$.

Let

$$
\begin{aligned}
& T_{1}=A_{i k} B_{j l}-B_{j l} A_{i k} \\
& T_{2}=A_{j k} B_{i l}-B_{i l} A_{j k} \\
& T_{3}=B_{j l}^{*} B_{j l}+A_{i k}^{*} A_{i k} \\
& T_{4}=B_{i l}^{*} B_{i l}+A_{j k}^{*} A_{j k} .
\end{aligned}
$$

Hence, $T_{1}=T_{2}, R a n T_{1} \subseteq R a n T_{3}, R a n T_{2} \subseteq R a n T_{4}$.

We claim that $T_{4} T_{3}=0$. Then $\operatorname{Ran}\left(T_{3}\right) \subseteq \operatorname{Ker}\left(T_{4}\right)$. But $R a n T_{1} \subseteq R a n T_{3}$ will imply that $\operatorname{RanT}_{1} \subseteq \operatorname{Ker} T_{4}$. Hence, $\operatorname{Ran}\left(T_{2}\right) \subseteq \operatorname{Ker}\left(T_{4}\right)=\overline{\operatorname{Ran}\left(T_{4}^{*}\right)}=$ ${\overline{\operatorname{Ran}\left(T_{4}\right)}}^{\perp}$. But $\operatorname{Ran}\left(T_{2}\right) \subseteq \operatorname{Ran}\left(T_{4}\right)$, which implies that $\operatorname{Ran}\left(T_{2}\right)=0$ and hence that both $T_{2}$ and $T_{1}$ are zero. Thus, the proof of the lemma will be complete if we can prove the claim

$$
\begin{gathered}
T_{4} T_{3}=\left(B_{i l}^{*} B_{i l}+A_{j k}^{*} A_{j k}\right)\left(B_{j l}^{*} B_{j l}+A_{i k}^{*} A_{i k}\right) \\
=B_{i l}^{*} B_{i l} B_{j l}^{*} B_{j l}+B_{i l}^{*} B_{i l} A_{i k}^{*} A_{i k}+A_{j k}^{*} A_{j k} B_{j l}^{*} B_{j l}+A_{j k}^{*} A_{j k} A_{i k}^{*} A_{i k} .
\end{gathered}
$$

From Lemma 1.3, we have $\forall i \neq j, B_{i l} B_{j l}=0$, implying $B_{i l} B_{j l}^{*}=0$ as $B_{j l}$ is a normal partial isometry. 
Again, from Lemma 1.4 $\forall k \neq l, B_{i l} A_{i k}=0$. Then $A_{i k}$ is a normal partial isometry implies that $B_{i l} A_{i k}^{*}=0 \forall k \neq l$.

Similarly, by taking the adjoint of the relation $B_{j l} A_{j k}^{*}=0 \forall k \neq l$ obtained from Lemma 1.4 we have $A_{j k} B_{j l}^{*}=0$.

From Lemma 1.3. we have $A_{j k} A_{i k}=0 \forall i \neq j . A_{i k}$ is a normal partial isometry implies that $A_{j k} A_{i k}^{*}=0 \forall i \neq j$.

Using these, we note that $T_{4} T_{3}=0$, which proves the claim and hence the lemma.

\section{Lemma 1.9.}

$$
\begin{aligned}
& A_{i k} B_{j k}=0=B_{j k} A_{i k} \\
& A_{k i} B_{k j}=0=B_{k j} A_{k i}
\end{aligned}
$$

$\forall i \neq j$ and $\forall k$.

Proof. By Lemma 1.3, we have $A_{i k} B_{j k}=0$ and $B_{j k} A_{i k}^{*}=0 \forall i \neq j$. The second relation along with the fact that $A_{i k}$ is a normal partial isometry implies that $B_{j k} A i k=0 \forall i \neq j$.

Thus, $A_{i k} B_{j k}=0=B_{j k} A_{i k} \forall i \neq j$.

Applying $\kappa$ to the above equation and using that $B_{k j}$ and $A_{k i}$ are normal partial isometries, we have $A_{k i} B_{k j}=0=B_{k j} A_{k i}$.

Lemma 1.10. $A_{i k} B_{i k}=B_{i k} A_{i k} \forall i, k$.

Proof. We have $A_{i j}^{*} B_{i j}=0=B_{i j}^{*} A_{i j}$ from Lemma 1.2. Using the fact that $B_{i j}$ and $A_{i j}$ are normal partial isometries we have $A_{i j}^{*} B_{i j}^{*}=0=B_{i j}^{*} A_{i j}^{*}$ and hence $A_{i j} B_{i j}=B_{i j} A_{i j}$.

Lemma 1.11. $A_{i k} A_{j l}=A_{j l} A_{i k} \forall i \neq j, k \neq l$.

Proof. Using (18) in Lemma 1.7 we proceed as in Lemma 1.8 to get $\operatorname{Ran}\left(A_{i k} A_{j l}-\right.$ $\left.A_{j l} A_{i k}\right) \subseteq \operatorname{Ran}\left(A_{j l} A_{j l}^{*}+A_{i k} A_{i k}^{*}\right)$ and $\operatorname{Ran}\left(A_{j k} A_{i l}-A_{i l} A_{j k}\right) \subseteq \operatorname{Ran}\left(A_{i l} A_{i l}^{*}+\right.$ $\left.A_{j k} A_{j k}^{*}\right)$.

We claim that $\left(A_{i k} A_{i k}^{*}+A_{j l} A_{j l}^{*}\right)\left(A_{j k} A_{j k}^{*}+A_{i l} A_{i l}^{*}\right)=0$.

Then by the same reasoning as given in Lemma 1.8 we will have $A_{j k} A_{i l}=A_{i l} A_{j k}$.

To prove the claim, we use $A_{i k} A_{j k}=0 \forall i \neq j$ from Lemma 1.3 (which implies $A_{j k}^{*} A_{i k}=0 \forall i \neq j$ as $A_{i k}$ is a normal partial isometry), $A_{i l}^{*} A_{i k}^{*}=0 \forall k \neq l$ from Lemma 1.4 (which implies $A_{i l}^{*} A_{i k}=0 \forall k \neq l$ as $A_{i k}$ is a normal partial isometry) and $A_{i l} A_{j l}=0 \forall i \neq j$ from Lemma 1.3 (which implies $A_{j l}^{*} A_{i l}=0 \forall i \neq j$ as $A_{i l}^{*}$ is a normal partial isometry).

Lemma 1.12.

$$
\begin{aligned}
A_{i k} A_{i l} & =A_{i l} A_{i k} \forall k \neq l \\
A_{i k} A_{j k} & =A_{j k} A_{i k} \forall i \neq j .
\end{aligned}
$$

Proof. From Lemma 1.3, we have $A_{k i} A_{l i}=0 \forall k \neq l$.

Applying $\kappa$ and taking the adjoint, we have $A_{i k} A_{i l}=0 \forall k \neq l$. Interchanging $k$ and $l$, we get $A_{i l} A_{i k}=0 \forall k \neq l$. Hence, $A_{i k} A_{i l}=A_{i l} A_{i k} \forall k \neq l$.

From Lemma 1.3. we have $A_{i k} A_{j k}=0 \forall i \neq j$. Interchanging $i$ and $j$, we have $A_{j k} A_{i k}=0 \forall i \neq j$. 
Remark 1.13. Proceeding in a similar way, we have that the $B_{i j}$ 's commute among themselves.

Theorem 1.14. The quantum isometry group of $\mathbb{T}^{n}$ is commutative as a $C^{*}$-algebra and hence coincides with the classical isometry group.

Proof. The proof follows from the results in Lemmas 1.8-1.12 and the remark following them.

Corollary 1.15. Using Theorem 3.13 of [5], we conclude that the quantum isometry group of the noncommutative $n$-tori $\mathbb{T}_{\theta}^{n}$ is a Rieffel deformation of the quantum isometry group of $\mathbb{T}^{n}$.

\section{ACKNOWLEDGEMENT}

We thank an anonymous referee for mentioning the paper [9], where A. Van Daele removed Woronowicz's separability assumption (in [7) for the $C^{*}$-algebra of the underlying compact quantum group.

\section{REFERENCES}

[1] Banica, T.: Quantum automorphism groups of small metric spaces, Pacific J. Math. 219(2005), no. 1, 27-51. MR2174219 (2006h:16054)

[2] Banica, T.: Quantum automorphism groups of homogeneous graphs, J. Funct. Anal. 224(2005), no. 2, 243-280. MR2146039 (2006d:16061)

[3] Connes, A.: Noncommutative Geometry, Academic Press, London-New York (1994). MR.1303779 (95j:46063)

[4] Goswami, D.: Quantum Group of Isometries in Classical and Noncommutative Geometry, Comm. Math. Phys. 285(2009), no. 1, 141-160. MR2453592

[5] Goswami, D.; Bhowmick, J.: Quantum Isometry Groups: Examples and Computations, Comm. Math. Phys. 285(2009), no. 2, 421-444. MR2461983

[6] Wang, S.: Quantum symmetry groups of finite spaces, Comm. Math. Phys. 195(1998), 195211. MR 1637425 (99h:58014)

[7] Woronowicz, S. L.: Compact quantum groups, pp. 845-884 in Symétries quantiques (Quantum symmetries) (Les Houches, 1995), edited by A. Connes et al., North-Holland, Amsterdam, 1998. MR $1616348(99 \mathrm{~m}: 46164)$

[8] Maes, Ann; Van Daele, Alfons: Notes on Compact Quantum Groups. Niew Arch. Wisk. (4) 16(1998), no. 1-2, 73-112. MR1645264 (99g:46105)

[9] Van Daele, Alfons: The Haar measure on a compact quantum group, Proc. Amer. Math. Soc. 123(1995), 3125-3128. MR.1277138 (95m:46097)

[10] Soltan, P. M.: Quantum families of maps and quantum semigroups on finite quantum spaces, preprint, arXiv:math/0610922.

[11] Woronowicz, S. L.: Pseudospaces, pseudogroups and Pontriagin duality, Proceedings of the International Conference on Mathematical Physics, Lausanne (1979), Lecture Notes in Physics 116, Springer, Berlin-New York, 1980, pp. 407-412. MR582650(82e:46079)

Stat-Math Unit, Kolkata Centre, Indian Statistical Institute, 203, B. T. Road, Kolkata 700 108, INDiA

E-mail address: jyotish_r@isical.ac.in 